\title{
El planteo heideggeriano sobre la inapariencia en el Seminario de Zähringen: \\ "Diese Phänomenologie ist eine Phänomenologie des Unscheinbaren"
}

\author{
HERNÁN INVERSO* \\ Universidad de Buenos Aires - ANCBA/CONICET (Argentina) \\ hernaninverso@conicet.gov.ar
}

\begin{abstract}
Resumen
Numerosas derivas de la fenomenología contemporánea, especialmente francesa, tematizaron fenómenos con excedencia que desafían la estructura husserliana de correlación intencional e impronta epistémica del polo subjetivo. La filosofía heideggeriana se pregunta sobre la facticidad y sus fundamentos, arribando al terreno en que el fenómeno se sustrae y resulta, por ello, inaparente, pero a la vez fundante de todo el ámbito del aparecer. En el presente trabajo estudiaremos en primer lugar el marco en que la filosofía de Heidegger tematiza la excedencia en el Seminario de Zähringen, consideraremos la tensión con la posición husserliana y analizaremos el planteo de la noción de "fenomenología de lo inaparente" para trazar algunas proyecciones de esta idea que habilitan la inclusión de las temáticas ligadas con la excedencia dentro de la economía propiamente fenomenológica.
\end{abstract}

Palabras clave: fenomenología, inapariencia, excedencia, donación.

\section{The Heideggerian approach on the inapparence in the Zäbringen Seminar: \\ "Diese Phänomenologie ist eine Phänomenologie des Unscheinbaren".}

\begin{abstract}
Many lines of contemporary phenomenology, especially in its French sides, have studied phenomena that exceed the limits and defy the original Husserlian structure and its commitment with intentional correlation and the epistemic aspects of the subjective pole. The direction of Heideggerian philosophy and its treatment about facticity and its foundations was a defining issue. In this context it is studied the phenomenon that subtracts itself and is, therefore, inapparent, but at the same time it is the founder of the whole field of appearance. The present work will study the framework in which Heidegger's philosophy thematizes excess in the Seminar of Zähringen. It will be considered the tension with the Husserlian position and the proposal of the notion of "phenomenology of the inapparent". On this basis it will be possible to draw some projections of this idea that enable the inclusion of the issues linked with exceedance within the phenomenological sphere.
\end{abstract}

Key words: Phenomenology, inapparency, exceedance, donation.

* Doctor en Filosofía, Docente de la Facultad de Filosofía y Letras de la Universidad de Buenos Aires (Argentina) e investigador de la Academia Nacional de Ciencias de Buenos Aires y el CONICET. Es autor de publicaciones en el área de fenomenología, entre las que se cuentan los libros El mundo entre paréntesis. Una arqueología de las nociones de reducción y corporalidad (2015) y Fenomenología de lo inaparente (2018). 
Heidegger produjo un desarrollo de ciertos campos asociados directamente con la donación y la excedencia que atraviesan todo el planteo fenomenológico. Muchos de ellos son planteados en una relación polémica con los estudios fenomenólogicos de tipo husserliano, que en esta lectura quedan asociados con los estudios estáticos y genéticos omitiendo los elementos que en estas esferas señalan claramente hacia un plano más allá de lo egológico ${ }^{1}$. En este punto cobra peso la noción de Gegebenheit, que se ha visto como elemento de la redefinición de la noción de verdad que lleva adelante Heidegger en el alejamiento de sus orígenes en la fenomenología husserliana.

En efecto, los tratamientos de Heidegger ofrecen una base para este diagnóstico, que exploraremos en lo que sigue considerando los desarrollos del último seminario dictado en 1973 en su casa de Friburgo, ubicada en el barrio de Zähringen. Prestaremos atención en primer lugar al espectro en el que surge el planteo sobre la relación entre excedencia e inapariencia, para estudiar luego los puntos explícitos de tensión con la posición husserliana y finalmente la propuesta de desarrollo de una fenomenología orientada a lo inaparente que resultará programática para las líneas posteriores. El recorrido de este trabajo apunta, por tanto, a relevar las bases y puntos de partida que han posibilitado el despliegue de estudios sobre la excedencia y son, por tanto, condición del planteo acerca de una fenomenología de lo inaparente a la que nos referiremos en el apartado final y provee elementos para acceder al arco completo de la fenomenicidad en sus derivas de estaticidad, geneticidad, generatividad e inapariencia a través de dispositivos diferentes que no dejan convivir la intencionalidad y la excedencia.

\section{EL HORIZONTE DEL PLANTEO SOBRE EXCEDENCIA E INAPARIENCIA}

La tematización de la excedencia como elemento que pone en cuestión la perspectiva husserliana asociada con el primado de la estructura intencional aparece a menudo como un instrumento crítico por parte de autores que pretenden tomar distancia, invertir o superar aspectos de la fenomenología en su instanciación originaria ${ }^{2}$. No son pocos, sin embargo, los contextos en que Husserl alude a la excedencia en su tematización de la intencionalidad dirigida a lo ausente. Tras el campo perceptivo se despliega un mundo latente con aspectos de cognoscibilidad a partir de la experiencia pasada e incognoscibilidad que "se extiende hasta abarcar el

1 Véase Steinbock (1995, 1998, 2003) e Inverso (2016, 2017) sobre estratos más allá de la estaticidad y la geneticidad.

2 Sobre esta práctica asociada con la filosofía de J.-L. Marion, véase Vinolo (2014). 
mundo como horizonte universal"3. El fondo indiferenciado adviene en los dos polos intencionales, dado que el objeto se muestra con lados ausentes y la conciencia se proyecta en experiencias retencionales y protencionales en un presente en el que opera la anonimidad del "proto-yo". Fenómeno sobreabundante y sujeto desbordado no son, por tanto, aspectos ajenos al universo de la fenomenología husserliana.

Dentro de la inapariencia habría que contar igualmente la dimensión de la teleología que remite a lo divino (Walton, 2011: 187), ${ }^{4}$ así como la serie de fenómenos caracterizados por su inaccesibilidad, detectados precisamente en la imposibilidad de fenomenización que quedan como fenómenos límite, en tanto se dan de modo inescindible con su incapacidad de darse e incluyen lo inconciente, el sueño, el nacimiento y la muerte, la temporalidad, el otro, los otros mundos, la vida animal y vegetal, la tierra, Dios, etc. (Steinbock, 2003: 290). Además de la 'generatividad' orientada a los elementos intrahistóricos, se despliega un Absoluto originario, en tanto si a la generatividad pertenece lo histórico, la dimensión de excedencia que adviene al explorar el acontecimiento no puede estar en un mismo plano sino en otro distinto. La excedencia se liga de este modo con la dimensión de la donación como fuente de toda mostración poniendo en primer plano con rasgos de inabarcable.

A pesar de esto, la práctica de priorizar los estudios asociados con los estratos estático y genético componen una visión de la fenomenología husserliana parcelada en sus alcances. No es casual que las obras de Michel Henry o Jean-Luc Marion, por nombrar dos ejemplos, multipliquen referencias a obras como Ideas I o La idea de la fenomenología procediendo a inferir enunciados generales sobre un constructo que difícilmente pueda sintetizarse en alguna de estas producciones tempranas. Una escala importante para el despliegue de estas posiciones está dado por los recorridos de la fenomenología heideggeriana y su temprano apartamiento en busca de una ontología del Dasein. Especialmente a partir de la estancia en Marburgo se profundiza una brecha ya presente donde Heidegger reniega de

\footnotetext{
3 Walton (2008: 169-187; 2010: 127-140; 2011: 187) y Rizo Patrón (2009; 2012: 381 395).

$4 \quad$ Esto surge de afirmaciones como: "Pero sí creo y hago consciente esta creencia, la efectúo libremente a partir de esta fuente práctica, entonces ella da sentido al mundo y a mi vida, proporciona la regocijada confianza (die freudige Zuversicht) en que nada es en vano y todo es para el bien" (Husserl, 1959: 355) y "Para creer en mí mismo y en mi verdadero yo, y en el desarrollo en esta dirección, debo creer en Dios, y en la medida en que hago esto, veo la guía divina, el consejo de Dios, la exhortación divina en mi vida" (Manuscrito A V, 21, 24b).
} 
la pretensión de certeza científica en aras de la comprensión de la existencia, como testimonian los desarrollos de Introducción a la investigación fenomenológica (Heidegger, 2006: 70-82).

Esa vía lleva progresivamente a profundizar en la tematización de una dimensión que no se reduce a lo fenoménico. En esta línea, en el influyente trabajo "El concepto de verdad en Husserl y Heidegger", E. Tugendhat sugirió la posibilidad de interpretar la posición heideggeriana como una radicalización producida precisamente a partir de la noción de Gegebenheit (Tugendhat, 1970, 1994). Para Husserl la fenomenología tendría por meta el esclarecimiento de la verdad que surge del análisis de los modos de donación, de modo que la asociación entre verdad y donación debería ser considerada como la herencia más férrea de un pensador al otro. Por su parte, Heidegger habría producido, a juicio de Tugendhat, una modificación que se concreta en el abandono de la intencionalidad, de modo tal que la donación adviene como correlato del revelar y la Lichtung como el proceso alumbrador (Garrido Periñán, 2015: 14-98). La ruptura puede remontarse, por tanto, a los resquicios por los que adviene la excedencia irreductible al esquema intencional, con un polo objetivo sobreabundante y un polo subjetivo desbordado.

En esta lectura, entonces, la reconsideración del concepto de donación provocaría una revisión de su postura, apelando al cuestionamiento de la correlación intencional y la función del polo subjetivo, de modo que se confiere al fenómeno una prioridad que en el modelo originario le estaría vedada ${ }^{7}$. Comprendido de este modo, el camino heideggeriano resulta un claro antecedente de las vertientes fenomenológicas que cuestionan la radicalidad de la versión husserliana sosteniendo que su compromiso con la intencionalidad se torna un obstáculo para una legítima consideración de la donación, dado que la restringe atendiendo sólo a la mostración según la lógica de la objetualidad y bloqueando toda manifestación de excedencia.

Es preciso enfatizar que estas derivas no se corresponden con una incapacidad de la fenomenología husserliana de dar cuenta de la donación en relación con fenómenos con excedencia, si bien es cierto que los desarrollos más numerosos sobre este terreno en la obra de Husserl se concentran en los últimos años y no han faltado estudiosos que sugieran un

Sobre este período de Heidegger, véase Godina (2011: 115-131).

Sobre este desarrollo, véase Smith (2007: 156-179), y Duits (2007: 207-223).

Sobre las limitaciones de esta idea, véase el apartado 1. 
incentivo en la competencia con las obras de Heidegger en estas direcciones del pensamiento husserliano (Bruzina, 1996: X) ${ }^{8}$. Heidegger puede haber estado tras el desarrollo de estos puntos y su tratamiento sostenido de estas cuestiones alimentó el crecimiento de otras líneas preocupadas sobre los alcances de lo fenoménico?.

En algún sentido toda la obra de Heidegger brinda elementos para estudiar este recorrido, pero hacia el final, en un clima de análisis retrospectivo, encontramos material valioso para acceder al modo en que Heidegger evaluaba la relación teórica con el suelo husserliano. En efecto, en 1973 Heidegger dictó el Seminario de Zähringen donde plantea una síntesis que resultaría de fundamental importancia para el desarrollo de la fenomenología posterior. Las discusiones asociadas con el llamado giro teológico de la fenomenología francesa contemporánea no dudan en remitir sistemáticamente a este texto como elemento disparador para la legitimación de esta línea (Janicaud, 1991; Restrepo, 2010; Inverso, 2013). No se trata, por cierto, de una relación de causación, dado que estas ideas, tanto en el terreno heideggeriano como en el de las innovaciones rupturistas ligadas con el giro teológico ya estaban en marcha, pero el poder de síntesis sobre la categoría en la que se insertarían estas líneas en el concierto general de la historia de la fenomenología puede sin dudas remitirse a este texto de Heidegger. Se trata, en efecto, de un texto de suma relevancia para la tematización de la excedencia por parte de autores posteriores y amerita por tanto una atención especial.

El planteo general se refiere a la cuestión del ser y su vínculo con tratamientos de Husserl, mostrando desde el inicio las dos temáticas centrales. Dos preguntas de Jean Beaufret acerca de la relación entre ambos guían la exploración. Por un lado, en qué medida se puede decir que en Husserl no hay pregunta por el ser, por otro, en qué sentido el análisis del mundo circundante es, en términos de Ser y Tiempo, un "logro esencial", dado que en otro lugar se lo considera secundario ${ }^{10}$. La relación entre ambos pensadores y la tensión entre la dirección que cada uno da a su obra queda, por tanto, en primer plano.

A propósito de la segunda pregunta Heidegger cifra el logro en el sentido de que por primera vez se tematiza la mundanidad del mundo y el ser-

\footnotetext{
8 La carta de Husserl a R. Ingarden en que se lamenta de que el público esté alemán esté tomado por "la moda pasajera de una filosofía de la "existencia" y "el abandono de la 'filosofía como ciencia rigurosa"' sugieren esta tensión (Husserl, 1994: III/II, 262).

9 Sobre el episodio de la publicación del texto de G. Misch en que se comparan las filosofías de Husserl y Heidegger, véase Sandmeyer (2009: 11-18).

10 Heidegger, 1977: 463. El otro lugar corresponde a "De la esencia del fundamento" (Heidegger, 1976: 155). Sobre Heidegger y Beaufret, véase Rafoul \& Jacerme (2002: 387 402) y Jacerme (2008).
} 
en-el-mundo es tomado como hecho primario, pero se torna secundario ante la pregunta por el sentido del ser y es por ello un medio, de modo tal que los $\int 14$ al 24 de Ser y Tiempo no son aislables del propósito general (Heidegger, 1986: 373). Este presupuesto ilumina la respuesta acerca de su relación con Husserl, donde comienza por notar que la pregunta por el sentido del ser apunta a su verdad, a la Wabrbeit des Sein, donde Wabrbeit implica la salvaguarda del ser como ser (Heidegger, 1986: 375). La puntualización persigue el propósito de afirmar que Husserl no tendría lugar en esta perspectiva, aunque la noción de "intuición categorial" en la sexta de las Investigaciones lógicas se acerca a este planteo (Heidegger, 1986: 376) ${ }^{11}$. En este punto encuentra Heidegger una referencia a la excedencia como Überschuss, en tanto al comprender algo como objeto adviene entre las afecciones sensibles un excedente que señala la presencia a través de un "es" que no es agregado sino algo visto "de otro modo que lo que es visible", y para que este tipo de visión acaezca debe ser dado. Hay un modo de darse categorial que es análogo de la intuición sensible y abre toda la dimensión de los fenómenos inaparentes.

No se trata, por supuesto, de una idea aislada. Los tratamientos de Heidegger sobre lo inaparente son múltiples e incluyen claramente el tratamiento del ocultamiento del ser imprescindible para pensar su desocultamiento. Estas derivas se profundizan, por cierto, en tanto Heidegger incorpora la noción de Ereignis para dar cuenta de aquello que hace posible que hombre y ser se apropien y pertenezcan uno al otro (Heidegger, 2000: 230) ${ }^{12}$. El Ereignis no es hombre ni ser, sino lo que se da en medio y se muestra como lo que destina las figuras del ser y se da ser, todo lo cual hace del ser un modo del Ereignis en tanto el que hace venir a la presencia. El Ereignis emerge, entonces, como trasfondo del ser y es a quien esencialmente se aplica el ocultarse, por lo cual afirma Heidegger que "el Ereignis es lo más inaparente de lo inaparente, lo más simple de lo simple" (Heidegger, 1985: 247 ${ }^{13}$, es la donación misma en su expresión asubjetiva de Es gibt, 'hay'. Inapariencia hay también en la Lichtung, que presupone lo inaparente del ser frente al cual se abre una zona despejada diferenciada de la oscuridad. Sobre esta base los planteos heideggerianos despliegan otros modos de lo inaparente que reflejan distintas relaciones marcadas

11 Heidegger se retrotrae a la diferencia entre la intuición sensible, que señala la byle, los datos sensoriales, donde todavía no hay objeto, y la intuición categorial que "da al ver una categoría". Sobre este punto, véase Peñalver Gómez (1985) y Vigo (2014: 142-144).

12 Véase Berciano (1991), Xolocotzi (2005), Sheehan (2001) y Lee (2001).

13 Sobre los elementos conflictivos de esta noción, véase Basso (2014: 51-74). 
por el retiro y la ausencia ${ }^{14}$. El Ereignis constituye "lo más inaparente de lo inaparente" en su oscilación entre hombre y ser (Heidegger, 1985: 247) y su papel de "entre" en el contraste entre Dios y el hombre (Heidegger, 1989: 26). No sólo lo divino se da en la excedencia del ser que se sustrae, sino que la filosofía surge como respuesta a un ser humano instanciado en la excedencia de lo presente donde Dios, ser y Ereignis tienen una condición inaparente (Heidegger, 1986: 331).

En este contexto ofrece Heidegger un eco antiguo que remonta a la figura de Antístenes, que se resistía a la teoría platónica de las Formas con la fórmula "veo el caballo, pero no la caballeidad"15. Lo hace para notar la distancia entre la captación de lo visible y la captación de una inapariencia fundamental, de modo que "es la sustancialidad la que sin embargo en su inapariencia permite a lo que aparece aparecer. En este sentido, se puede incluso decir que ella es más aparente que lo aparente mismo (ist es die Substan₹ialität, was in seinem Nichterscheinen dem Erscheinenden das Erscheinen ermöglicht. In diesem Sinne kann man sogar sagen, daß sie erscheinender als das Erschienene selbst ist)" (Heidegger, 1986: 377). Lo aparente, entonces, se funda en la inapariencia, surge sobre un fondo de inapariencia y para Heidegger "tal es el aporte decisivo de Husserl (So steht es um den entscheidenden Beitrag Husserls)" (Heidegger, 1986: 367).

Esta relevancia se plantea sobre el horizonte de la "caída" del pensamiento de Occidente, donde tras un inicio luminoso el ser queda preso de la noción de verdad como adecuación, es decir preso del juicio. El acercamiento husserliano y su planteo de la donación originaria habría roto con esta cautividad. Desde la perspectiva del proyecto heideggeriano, Husserl ofrece un ser que no es un mero concepto y señala hacia una pregunta que no llega a expresar porque entiende ser como ser-objeto. En este sentido, Heidegger despliega el mecanismo que guiará las preguntas por la inapariencia, en tanto indica la necesidad de distinguir 'ser objeto' de 'ser'. Con este dispositivo preanuncia la operación henryana, que apunta a distinguir 'aparecer' de 'aparecer del aparecer'. Cabe notar que esta deriva traza una distancia entre ser y aparecer que privilegia este último, con lo cual se subordina explícitamente la ontología a la fenomenología y se evita la caída en la metafísica en la cual Nietzsche y Heidegger cifraron el fracaso de la filosofía occidental ${ }^{16}$.

14 Sobre estos otros modos asociados con la relación entre mundo y cosas, al pertenecerse recíproco de los ámbitos del mundo, a la ocultación de Dios y al retirarse de las cosas, véase Walton (2011: 192-196).

15 Sobre la importancia de la figura de Antístenes para la comprensión de la ontología antigua, véase Brancacci (1990) e Inverso (2011), donde se sugieren proyecciones contemporáneas de los planteos del socrático.

16 Sobre este punto, véase Walton (2008) y Fernández Beites (2011: 35). 
A propósito de esto Heidegger señala que los tratamientos de Platón y Aristóteles condicionan toda deriva posterior asociando el ser con la $p a-$ rousía, la presencia: "toda la historia de la metafísica se caracteriza, por tanto, como historia del ser del ente (Alle Geschichte der Metaphysike erweist sich also als Geschichte des Seins des Seienden)" (Heidegger, 1986: 379), lo cual provoca que habiendo tratado siempre del ser nunca se haya preguntado por su sentido. Para ello es preciso invertir el programa husserliano y poner la conciencia entre paréntesis para que advenga el Dasein en familiaridad directa con el mundo. En este sentido, la conciencia y su remisión al "saber porque se ha visto" constituye un eco del eidénai griego en el Dasein, que mienta el estar en apertura, como die Lichtungsein y su remisión a ser el claro que funda la luz que permite el haber visto de toda conciencia (Heidegger, 1986: 380).

\section{EL ABANDONO DEL HORIZONTE HUSSERLIANO}

Más allá del reconocimiento de estos logros, el proyecto de inversión respecto de la posición de Husserl está sintetizado en el reconocimiento de ciertos elementos relevantes de la inapariencia, pero indicando que tal reconocimiento es incompleto e impide avanzar dado que la fenomenología husserliana incorpora la herencia cartesiana que hace de la subjetividad un obstáculo para la pregunta por el ser. De esta manera la intencionalidad otorga un lugar al objeto sólo en la inmanencia de la conciencia. La adopción del cogito compromete con una conciencia monádica que funciona a la manera de una caja y "la idea de 'salir' de esta caja es contradictoria en sí misma (Die Vorstellung, aus diesem geschlossenen Raum 'berauszukommen', ist in sich widersprüchlich)" (Heidegger, 1986: 383). No se trata sino de la hipostasiación de la vía cartesiana y una atención explícita a las Meditaciones Cartesianas, sin reparar en el resto del dispositivo husserliano ${ }^{17}$.

Las quejas que dejó testimoniadas Husserl en sus anotaciones marginales a Sery Tiempo donde ve desvirtuada su posición podrían ser trasladadas a estas afirmaciones del Seminario de Zähringen (Sheehan \& Palmer, 1997), dado que se construye el perfil de una fenomenología en riesgo de solipsismo a los efectos de contrastarlo con el de una hermenéutica fenomenológica "fuera de la caja" que "ve tinteros" sin datos hyléticos ni categorías y se abre por eso a "una experiencia fundamental de la cosa misma (Es kommt darauf an, eine Grunderfahrung des Dinges selbst zu machen)" (Heidegger, 1986: 383). Los ejemplos que despliega funcionan como eco de las primeras tomas de distancia sistemáticas que Heidegger había trazado en

17 Sobre las vías, Kern (1977), Geniusas (2012), Luft (2004), Staiti (2012) e Inverso (2016b). 
los inicios de su obra. El curso de 1919, La idea de la filosofía y el problema de la concepción del mundo, visto a menudo como la bisagra tras la que surgen las primeras críticas a Husserl asociadas con los rasgos que explicitan la vida preteórica como tema y la necesidad de un nuevo método de corte hermenéutico, ilustran su posición con ejemplos similares. En ese caso no es un tintero sino un mueble, una cátedra, y luego un libro (Heidegger, 1992: 71-73), donde se insiste en que los horizontes de significatividad y precomprensión son primarios, de modo que un libro nunca es una serie de páginas numeradas con manchas de tinta, sino siempre un libro en el plexo de sentido que lo contiene. Se trata, en efecto, de un proyecto de cumplimiento de la fórmula zu den Sachen selbst que atiende a los fenómenos con la estrategia de origen en el Dasein y no en la conciencia.

Volviendo al planteo del Seminario, Heidegger recuerda que el Dasein es caracterizado en Ser y Tiempo por el ék-stasis, componiendo un acercamiento como "ser ek-státicamente el ahí (das Da ek-statisch sein)" que privilegia la referencia al afuera y rompe toda inmanencia (Heidegger, 1986: 383). Esta ruptura de la inmanencia está presentada por Heidegger como una radicalización de la intencionalidad que, podríamos decir, invierte su sentido y configuraría la inversión focal que sustenta la inversión general del enfoque. Esta radicalicación de la intencionalidad lleva a decir que conduce al reconocimiento de que la conciencia se funda en el Dasein, en tanto se sitúa en lo ek-stático del Dasein, ék-stasis que refiere a la "instancia en el claro (Inständigkeit in der Lichtung)", que indica el centro de los tres ékstasis y la persistencia del ser. Con el comienzo desde el Dasein se asegura el estar "ekstáticamente frente a frente con lo que es en sí mismo (ek-statisch dem, was etwas selber ist, von Angesicht zu Angesicht)" y no con una representación fantasmática (Heidegger, 1986: 384).

La resistencia que pretende crear Heidegger queda retratada en la mención de la figura de F. Brentano, a quien reclama como su iniciador en la filosofía, de la misma manera que lo habría sido de Husserl. De esta manera corrige la filiación que lo coloca como discípulo de Husserl y redefine su posición respecto de sus influencias filosóficas como una línea en paridad con la husserliana. Así, su proyecto no es sólo una inversión a posteriori sino también una deriva paralela que remonta a un mismo origen. La imagen que sustenta esta idea es la de los "dos" Brentanos, el de Husserl, identificado con la Psicología desde el punto de vista empirico, y el suyo, que remite a Los múltiples sentidos del ente en Aristóteles. Se ve a simple vista que la 
descripción ilustra la distinción entre orientación a la conciencia y orientación al Dasein en familiaridad con el mundo que acaba de explicitarse (Heidegger, 1986: 385) ${ }^{18}$.

Husserl habría visto en Brentano la estrategia de recurso a la conciencia y la estructura de la intencionalidad, mientras Heidegger habría encontrado la homonimia del ser y su problemática unidad. La vuelta a los rasgos griegos del pensamiento surge en este punto como signo de una vía alternativa a la modernidad y sus ecos husserlianos. Así dirá Heidegger que se debe dejar atrás el objeto y su compromiso con la representacón para remontarse al mundo de cosas que entran en presencia, en parousia, que hablan desde el pasado griego. "El Brentano de Aristóteles" recuerda esos ecos y salva al joven Heidegger de los errores de la modernidad.

Notemos que el texto comienza con una imagen de Husserl que señala como aporte la tematización de la excedencia y avanza de manera cada vez más oscura en la denuncia de iteración de los equívocos modernos. Las dos cosas se conjugan en el perfil que se intenta delinear y eso explica que se pueda plantear una radicalización que es a la vez una vuelta a los orígenes sobre los desarrollos husserlianos, que son una continuación de la priorización de la conciencia, pero a la vez muestran las grietas que llevan a su superación. Esta vuelta es radicalización porque "entendido como $D a$ sein, es decir a partir de lo ek-stático, el hombre no es sino saliendo de sí hacia eso por completo otro que él, que es el claro del ser (als Da-sein verstanden, das heißt, von der Ek-statik aus, der Mensch nur ist, indem er von sich bis zu jenem ganz anderen als er selbst kommt, das die Lichtung des Seins ist)" (Heidegger, 1986: 386).

La duplicidad pone de manifiesto la misma actitud de tensión que Heidegger tuvo respecto de Husserl desde el inicio. Cabe recordar que después de una colaboración inicial que hizo pensar a Husserl que había encontrado un heredero y continuador, se produjo un distanciamiento por parte de Heidegger que creció en lo teórico a medida que se oscureció silenciosamente la relación personal. En efecto, mientras Husserl confiaba en una tarea conjunta, Heidegger acopió malestares diversos. Las cartas de 1923 que Heidegger dirigió a Löwith y Jaspers retratan un ánimo alterado donde se conjuga la inseguridad ante un futuro incierto y la desconfianza respecto del apoyo de Husserl, con una voluntad casi obsesiva de superar los logros de su maestro mezclada con un marcado desprecio por su valor intrínseco. Heidegger no se priva de decir a Löwith que no hay nada filosófico en la obra de Husserl y que se convirtió en un personaje ridículo

18 Sobre la figura de Brentano en la tensión entre Husserl y Heidegger, véase Del Negro (1953: 571-585) y Moran (2000: 39-65). 
sosteniendo la fenomenología como una misión ficticia (Kisiel \& Sheehan, 2007: 372).

A pesar de que Husserl efectivamente intervino a favor de Heidegger para que accediera al cargo docente en Marburgo, la actitud general de este último está lejos de ser amistosa y no duda en lamentar que "quizás el viejo advierta en verdad que le estoy retorciendo el cuello, y entonces se acaba la expectativa de la sucesión" (Kisiel \& Sheehan, 2007: 372; sobre este punto, ver Xolocotzi, 2008). Sería mucho más tarde, hacia 1930, que Husserl se lamentaría de la soledad en la que había quedado y de la actitud de sus discípulos que lo consideraban un viejo pasado de $\operatorname{moda}^{19}$. Ni siquiera después de los conflictos de 1927 a propósito de la redacción del artículo de la Enciclopedia británica perdió Husserl confianza en Heidegger y procedió a asegurarle la ansiada sucesión en Friburgo, a pesar de las quejas de otros discípulos ${ }^{20}$.

Recordamos estos elementos dado que el texto que nos ocupa sintetiza el reconocimiento y la distancia que había marcado la relación entre ambos, en un momento en que ya había pasado mucho tiempo de los contactos personales y Heidegger cuenta con la perspectiva suficiente para dejar testimonio de su juicio sobre la relación entre ambas filosofías. No es menor que se trate del último seminario que dictó Heidegger, como tampoco lo es que el testimonio que queda deje entrever la persistencia de una relación conflictiva, donde el reconocimiento no termina de producirse del todo o convive, en todo caso, con la necesidad de dejar sentada la preeminencia de su propia posición.

\section{INAPARIENCIA Y PENSAMIENTO TAUTOLÓGICO}

El tramo final del planteo se desliza hacia el Gestell, el modo extremo de la historia de la metafísica, del destino del ser que revela la disponibilidad en la época que es época y pone el ser en epoché, entre paréntesis, de modo que ya no hay objetos sino bienes de consumo a disposición de consumidores en el mercado (Baruzzi, 1983; González Fisac, 2007). En la

\footnotetext{
19 En 1930 escribe Husserl a G. Albrecht: "Es una situación trágica, en la que, a pesar de que estoy absolutamente seguro de que en la última década llevé mi filosofía fenomenológica a la madurez, a la claridad y pureza, a un amplitud de problemas y métodos abarcativos que trazan el genuino sentido y la vía para la filosofía de todo el futuro, vino una nueva generación a escena que malinterpreta mis fragmentos y comienzos incompletos publicados en su sentido más profundo, que propaga una fenomenología supuestamente mejorada y me reverencia como a un viejo pasado. Así es que estoy otra vez filosóficamente solo, como estaba cuando empecé" (Husserl, 1994: 75).

20 Véase el caso de Pfänder en Husserl, 1994: 180-184.
} 
trama de estos imperativos, se trata de pensar al hombre a partir del $D a$ sein como posibilidad de relacionarse con el dominio de lo que no es el hombre. La reflexión sobre el Gestell no es ajena a las reflexiones sobre la fenomenología de lo inaparente, dado que el planteo heideggeriano se propone puntualizar que los imperativos que dominan el mundo humano se conjugan con un asedio de la naturaleza como proveedora y reserva de energía, de modo tal que recibe la misma violencia generalizada. El modo de dar cuenta de esta violencia se ilustra con una fórmula que remite a la expresión de los principios de la fenomenología: "a tanto desafío de la naturaleza, tanto desafío al que el hombre se somete (je größer die Herausforderung der Natur, umso größer die Herausforderung, der der Mensch selbst unterliegt)" (Heidegger, 1986: 391) ${ }^{21}$. Con eso se señala que en esta lógica el hombre queda atado al proyecto extractivo en una carrera de maximización y multiplicación de logros tecnológicos.

La entrada en el dominio del Dasein que se asocia con la instancia en el claro del ser remite a una vuelta a los inicios que Heidegger ejemplifica con la mirada hacia Parménides que debe superar "la torpeza y propiamente ingenuidad de la 'destrucción ontológica' (die Ungeschicklichkeit und strenggenommen Naivität der 'ontologischen Destruktion)" (Heidegger, 1986: 395). Esto provoca un rechazo de esta categoría de Ser y Tiempo en razón de detectar en ella un estadio primitivo de la historia del ser cuya contracara se propone ofrecer con la investigación que inmediatamente lleva a cabo sobre el verso del proemio del poema de Parménides que menciona el corazón de la verdad. Junto con Anaximandro y Heráclito, Parménides resulta un pensador inicial, es decir que tomó como punto de partida el ser (Heidegger, 1982: 10; véase Decker, 1981), y es por ello que del análisis del pasaje surge la caracterización de alétheia como des-ocultamiento del plano del ser.

Heidegger compara la dificultad de este acceso al ser con el modo en que Husserl vislumbra la noción de intuición categorial y su excedencia. Así es que la pregunta por el ser excede a los entes y se aproxima mediante el señalamiento de la relación entre eînai y entrar en presencia. La noción de ser señalaría, en este sentido, a un fundamento mostrativo que indica que detrás del ser está el aparecer, de modo tal que la fórmula ésti gàr eînai queda vertida como "presencia, en efecto, presenciar (anwest nämlich Anwesen)" y "presencia presenciar mismo (anwest Anwesen selbst)", cuya tautológica extrañeza se explica porque "estamos aquí en el dominio de lo inaparente (Wir sind hier im Bereich des Unscheinbaren)" (Heidegger, 1986: 397; véase Walton, 2009).

21 Sobre los principios, véase el tratamiento en M. Henry y J.-L. Marion y su conexión con Husserl en Inverso (2016a: 288-304). 
Se trataría, así, de un modo de advertir sobre las variaciones de legalidades que advienen necesariamente en el plano de la fenomenología de lo inaparente y por momentos contravienen los parámetros que rigen otros accesos. En nuestra lectura, esta advertencia debería matizar toda la lectura que Heidegger hace de Husserl, trazando más bien su idea como una insistencia en la necesidad de desplegar una exploración de la fenomenología de lo inaparente. Este despliegue implica dispositivos que, como hemos visto, Heidegger plantea y encontraremos potenciados y ampliados en las derivas que encontraron en esta tensión entre Husserl y Heidegger un ámbito propicio para ensayar inversiones que abren la atención a lo inaparente.

La idea de que estas lecturas implican inversiones, si bien no está presente el término, se remonta a esta interpretación heideggeriana, de modo que a ella tanto como a las líneas continuadoras se aplica la objeción de que el espacio para la excedencia en terreno husserliano es más amplio que la mera mención de la intuición categorial. En este sentido, es posible conjugar todas estas derivas dentro de un ámbito que no necesita explícitamente renegar de la perspectiva de la conciencia, sino que los enfoques esbozados y sus varios modelos de reducción pueden convivir en un proyecto de estudio fenomenológico abarcativo que intente dar cuenta de todo el arco de mostración del fenómeno.

El estudio del texto parmenídeo le permite a Heidegger caracterizar al ser como corazón de la verdad y afirmar que la alétheia no es una apertura vacía sino un desocultamiento que rodea a lo que es como presencia. La dimensión de inapariencia va unida, por tanto, a la idea de que estos rasgos no están sujetos a demostración, es decir que no se accede a este plano por vía epistémica. Para ello ofrece apoyo el verso 28 del fragmento 1 de Parménides (DK 28B1.28), traducido como "es necesario que hagas la experiencia de todo (nötig ist, dass du die Erfahrung von allem machst)", que indicaría "un fundarse en lo que ha aparecido a la mirada (ein Sich gründen auf das, was sich dem Blick hat sehen lassen)" (Heidegger, 1986: 399).

Este pensamiento es caracterizado como pensamiento tautológico, ajeno, por tanto, a los métodos de las ciencias ${ }^{22}$. Sostiene Heidegger a propósito de esto que este pensamiento, que en Qué significa pensar había caracterizado diciendo que "lo peculiar de las proposiciones de esta índole reside en que no dicen nada y a la vez vinculan el pensar del modo más decisivo a su asunto" (Heidegger, 2002: 157), es el que originariamente pertenece a la fenomenología. El marco de este pensar tautológico es el que sirve para decir que Das Ereignis ereignet, "el Ereignis acontece y apropia"

22 Sobre las implicancias del pensamiento tautológico, véase Courtine (1993), Scheier (1993: 60-74), Bassler (2001) y Roesner (2006). 
(Heidegger, 2007: 29) y representa la consecución de un camino, frente a los métodos que caracterizan a las ciencias. Encarna, de este modo, una distancia abismal entre la filosofía y la ciencia que obstaculiza diálogos posibles. Esto puede matizarse si se entiende que esta caracterización atiende a un modo peculiar de la fenomenología entendida como fenomenología de lo inaparente que no va en contra esencialmente de los demás modos asociados con la estaticidad, la geneticidad y la generatividad, que son modalidades con posibilidades más amplias de dialogar con las ciencias ${ }^{23}$.

En terreno husserliano la psicología puede ser una vía a la reducción, como puede serlo la antropología e incluso el mundo de la vida en general. Si de fenomenología de lo inaparente se trata, las peculiaridades se multiplican, lo cual no es otra cosa que lo que Heidegger afirma: "Así comprendida, la fenomenología es un camino que conduce allí, adelante, y deja mostrarse a aquello ante lo cual es conducido. Esta fenomenología es una fenomenología de lo inaparente (So verstanden ist die Phänomenologie ein Weg, der hin-führt vor. . und sich das zeigen läßt, wovor er geführt wird. Diese Phänomenologie ist eine Phänomenologie des Unscheinbaren)" (Heidegger, 1986: 399). Si entendemos esta formulación de modo tal que se está hablando efectivamente de una fenomenología de lo inaparente que no agota toda investigación fenomenológica, esta caracterización es un aporte valioso para dar cuenta precisamente de los rasgos que adquiere la fenomenología en este nivel más alto, donde se trata de "dejar mostrarse aquello ante lo cual es conducido". De este modo, contra muchos de sus críticos, la fenomenología no está presa de la economía de la objetualidad, identificada con los estratos estático y genético, sino que puede dar cuenta de fenómenos geohistóricos y culturales en el estrato de la generatividad y también puede enfrentar el desafío de la excedencia precisamente en el estrato de la fenomenología de lo inaparente comprendido como dispositivo para dar cuenta de los fenómenos límite.

\section{COROLARIOS}

El recorrido por este tratamiento que ha generado las discusiones recientes sobre excedencia e inapariencia y revela los mecanismos de la construcción heideggeriana de una tensión respecto de la posición de Husserl. Varios de ellos, como hemos notado, resultan recortes en cierto modo arbitrarios que omiten puntos importantes de la posición husserliana referidos a la reflexión sobre la excedencia. Con todo, el planteo resulta de

23 Sobre los estratos de la fenomenología, véase Steinbock (1995, 1998, 2003) a propósito de la generatividad, e Inverso (2016a, 2016b), respecto de un arco de cuatro estratos conectados. 
impacto y es efectivo en la tarea de llamar la atención sobre una región desatendida del arco de la fenomenicidad. Esta interpretación de la donación implicaría una redefinición de la horizonticidad husserliana, en tanto Husserl contempla la referencia al mundo como horizonte de todos los horizontes y en un mismo plano que ellos, mientras Heidegger los distancia identificando el horizonte con la determinación y dejando fuera de él un ámbito originario previo a la horizonticidad (Heidegger, 1995: 113). Queda pensar una alternativa a esta colisión, que surge de concebir la posibilidad de que un horizonte de todos los horizontes con indeterminación interna y no exterior, así como de una tematización de la donación misma que la comprenda como fuente transhorizóntica, en el sentido de que enfoca no la relación de los horizontes en el horizonte de todos los horizontes sino el aparecer del aparecer que hace posible los horizontes y se remonta, por tanto, a las regulaciones teleológicas últimas.

Así, los desarrollos heideggerianos que inspiraron las vertientes de fenomenología francesa que denuncian la falta de radicalidad de la posición de Husserl para proponer alternativas en la Vida, como M. Henry, o en una fenomenología de la donación, como J.-L. Marion, pueden asimismo apoyar un trabajo de relectura que funde el terreno de una fenomenología de lo inaparente integrado al resto de los espacios fenomenológicos. De este modo, estaticidad, geneticidad, generatividad e inapariencia ofrecen dispositivos diferentes y variados para encarar la captación de distinto tipo de fenómenos, desde el plano de la estructura hasta la excedencia última.

\section{REFERENCIAS}

-Baruzzi, A. (1983). Heidegger: Gestell und Gelassenheit. Allgemeine Zeitschrift für Philosophie, 8(2), 1-6.

-Bassler, O. (2001). The Birthplace of Thinking: Heidegger's Late Thoughts on Tautology. Heidegger Studies, 17, 117-133.

-Basso, L. (2014). Investigaciones acerca de la estructura conflictiva del Ereignis en Beiträge zur philosophie de Martin Heidegger. Thémata, 49, 51-74.

-Berciano, M. (1991) El evento (Ereignis) como concepto fundamental de la filosofía de Heidegger. En Acercamiento a la obra de Martin Heidegger (pp. 91118). Salamanca: Sociedad Castellano-Leonesa de Filosofía.

-Berciano, M. (2002). "Ereignis: la clave del pensamiento de Heidegger". Thémata, 28, 47-69.

-Courtin, J. (1993). Phenomenology and/or tautology. Reading Heidegger: Commemorations. Bloomington: Indiana University.

-Decker, J. (1981). Phenomenology of the Unapparent: Heidegger on Parmenides. Dissertation, Boston College.

-Del Negro, W. (1953). Von Brentano über Husserl zu Heidegger. Zeitschrift für philosophische Forschung, 7(4), 571-585. 
-Duits, R. (2007). On Tugendhat's Analysis of Heidegger's Concept of Truth. International Journal of Philosophical Studies, 15(2), 207-223.

-Garrido Periñán, J. (2015). Ser y Lichtung: Heidegger ante el claro del ser. Desafíos fenomenológicos en torno al claro del ser. Madrid: Liber.

-Geniusas, S. (2012). The Origins of the Horizon in Husserl's Phenomenology. London: Springer.

-Godina, L. (2011). Breves notas para entender el neokantismo marburgués de Heidegger. En Á. Xolocotzi et al. (Eds). Ámbitos fenomenológicos de la hermenéutica (pp. 115-131). México: Eón.

-González Fisac, J. (2007). El sueño del hombre: antropología y metafísica en Heidegger. Thémata, 39, 515-520.

-Heidegger, M. (1975-). Gesamtausgabe = GA. Frankfurt: Klostermann.

-Heidegger, M. (1976). Wegmarken (1919-1961), ed. F.-W. von Herrmann (GA 9), Frankfurt: Klostermann.

-Heidegger, M. (1977). Sein und Zeit (1927), ed. F.-W. von Herrmann (GA 2), Frankfurt: Klostermann.

-Heidegger, M. (1982). Parmenides (Winter semester 1942/43), ed. M. S. Frings (GA 54), Frankfurt: Klosterman.

-Heidegger, M. (1985). Unterwegs zur Sprache (1950-1959), ed. F.-W. von Herrmann (GA 12), Frankfurt: Klostermann.

-Heidegger, M. (1986). Seminare (1951-1973), ed. C. Ochwadt (GA 15), Frankfurt: Klostermann.

-Heidegger, M. (1989). Beiträge zur Philosophie (Vom Ereignis) (1936-1938), ed. F.W. von Herrmann (GA 65), Frankfurt: Klostermann.

-Heidegger, M. (1992). Zur Bestimmung der Philosophie (GA 56/57), Frankfurt: Klostermann.

-Heidegger, M. (1995). Feldweg-Gespräche (1944/45), ed. I. Schüssler (GA 77), Frankfurt: Klostermann.

-Heidegger, M. (2000). Vorträge und Aufsätz̧e (1936-1953), ed. F.-W. von Herrmann (GA 7), Frankfurt: Klostermann.

-Heidegger, M. (2002). Was heisst Denken? (1951-1952), ed. P.-L. Coriando (GA 8), Frankfurt: Klostermann.

-Heidegger, M. (2006). Einführung in die phänomenologische Forschung (Winter semester 1923/24), ed. F.-W. von Herrmann (GA 17), Frankfurt: Klostermann.

-Heidegger, M. (2007). Zur Sache des Denkens (1962-1964), ed. F.-W. von Herrmann (GA 14), Frankfurt: Klostermann.

-Husserl, E. (1950-). Gesammelte Werke-Husserliana I-LX = Hua. Dordrecht, Springer.

-Husserl, E. (1959). Erste Philosophie (1923/4). Zweiter Teil: Theorie der phänomenologischen Reduktion. Edited by Rudolf Boehm (Hua VIII). The Hague, Netherlands: Martinus Nijhoff, 1959.

-Husserl, E. (1994). Briefwechsel. Edited by Karl Schuhmann. The Hague, Netherlands: Kluwer Academic Publishers.

-Inverso, H. (2013). De E. Husserl a J.-L. Marion: donación y límites de la fenomenología. Franciscanum, LV(159), 127-154. 
-Inverso, H. (2014). 'El espíritu absoluto debería tener también un cuerpo'. Una revisión de la relación entre epoché y corporalidad en Husserl y Merleau-Ponty. Devenires, 29, 63-82.

-Inverso, H. (2015). El mundo entre paréntesis. Una arqueología de las nociones de reducción y corporalidad. Buenos Aires: Prometeo.

-Inverso, H. (2016). Fenomenología de lo inaparente. Buenos Aires: Universidad de Buenos Aires, Tesis doctoral.

-Inverso, H. (2017). La fenomenología de lo inaparente y el problema de las vías hacia el plano trascendental. Eidos, 26, 43-73.

-Inverso, H. (2018). ¿Qué es la fenomenología? La vía psicológica y la colaboración entre Husserl y Heidegger en el artículo de la Enciclopedia Británica. Daimon. Revista internacional de Filosofía, 78, 181-198.

-Jacerme, P. (2008). The Toughtful Dialogue Between Martin Heidegger and Jean Beaufret: A New Way of Doing Philosophy. En D. Pettigrew y F. Raffoul (Eds.), French Interpretations of Heidegger: An Exceptional Reception (pp. 59-72). New York: SUNY Press.

-Janicaud, D. (1991). Le Tournant théologique de la phénoménologie française. Combas, Éd. de l'Éclat.

-Kern, I. (1977). The Three Ways to the Transcendental Phenomenological Reduction in the Philosophy of Edmund Husserl. En F. Elliston et al. (Eds.) Husserl: Exposition and Appraisals (pp. 126-149). Notre Dame: University of Notre Dame Press.

-Kisiel, T., Sheehan, T. (Eds.) (2007). Becoming Heidegger. On the Trail of His Early Occasional Writings, 1910-1927, Evanston, Il., Northwestern University Press.

-Lee, S. (2001). Existenz und Ereignis: eine Untersuchung zur Entwicklung der Philosophie Heideggers. Wurzburg: Königshausen \& Neumann.

-Luft, S. (2004). Husserl's Theory of the Phenomenological Reduction: Between Life-World and Cartesianism. Research in Phenomenology, 34(1), 198-234.

-Moran, D. (2000). Heidegger's Critique of Husserl's and Brentano's Accounts of Intentionality. Inquiry, 43(1), 39-65.

-Peñalver Gómez, P. (1985). Entre Husserl y Heidegger: un equívoco inextricable. Anales de filosofía 3, 111-123.

-Raffoul, F. \& Jacerme, P. (2002). Martin Heidegger et Jean Beaufret: Un Dialogue. Revue Philosophique de la France Et de l'Etranger, 192(4), 387-402.

-Restrepo, C. (2010). El "giro teológico" de la fenomenología: Introducción al debate. Pensamiento y Cultura, 13(2), 115-126.

-Rizo Patrón, R. (2009). Más allá del solipsismo y la identidad: horizontes de descentramiento y excedencia en la fenomenología de Husserl. En ocasión del sesquicentenario del nacimiento de Husserl. Lima: PUCP.

-Rizo Patrón, R. (2012). Horizontes de descentramiento y excedencia. Tiempo, intersubjetividad, generatividad. Acta fenomenológica latinoamericana, 4, 381-395.

-Roesner, M. (2006). Heidegger, du Nous au Geist: De la tautologie. Heidegger et la question de l'esprit. Les Etudes philosophiques, 76(1), 63-88.

-Sandmeyer, B. (2009). Husserl's Constitutive Phenomenology: Its Problem and Promise. New York: Routledge. 
-Scheier, C. (1993). Die Sprache spricht. Heideggers Tautologien. Zeitschrift für philosophische Forschung, 47, 60-74.

-Sheehan, T. \& Palmer, R. (1997). Psychological and Transcendental Phenomenology and the Confrontation with Heidegger (1927-1931): The Encyclopaedia Britannica Article, The Amsterdam Lectures, "Phenomenology and Anthropology". Dordrecht: Springer.

-Sheehan, T. (2001). Kehre and Ereignis: A prolegomenon to introduction to metaphysics. En R. Polt \& G. Fried (Eds.), A companion to Heidegger's Introduction to metaphysics (pp. 3-17). New Haven: Yale University Press.

-Smith, W. (2007). Why Tugendhat's Critique of Heidegger's Concept of Truth Remains a Critical Problem. Inquiry, 50(2), 156-179.

-Staiti, A. (2012). The Pedagogic Impulse of Husserl's Ways into Transcendental Phenomenology. Graduate Faculty Philosophy Journal, 33(1), 39-56.

-Steinbock, A. (1995). Home and Beyond: Generative Phenomenology After Husserl. Illinois: Northwestern University Press.

-Steinbock, A. (1998). Husserl's Static and Genetic Phenomenology: Translator's Introduction to two Essays. Continental Philosophy Review, 31, 127-134.

-Steinbock, A. (2003). Generativity and the Scope of Generative Phenomenology. En D. Welton (Ed.), The New Husserl: A Critical Reader (pp. 289-325). Indiana: Indiana University Press.

-Tugendhat, E. (1970). Der wahrheitsbegriff bei Husserl und Heidegger. Berlin: de Gruyter.

-Tugendhat, E. (1994). Heidegger's Idea of Truth. En Brice R. Wachterhauser (Ed.), Hermeneutics and Truth (pp. 83-97). Illinois: Northwestern University Press.

-Vinolo, S. (2014). Le realisme spéculatif à l'épreuve de la donation. Théoremes 6.

-Walton, R. (2001). Fenomenología de la empatía. Philosophica, 24, 409-428.

-Walton, R. (2008). Reducción fenomenológica y figuras de la excedencia. Tópicos, $16,169-187$.

-Walton, R. (2009). El aparecer y lo latente. Acta fenomenológica latinoamericana, 3, 105-120.

-Walton, R. (2010). El giro teológico como retorno a los orígenes: La fenomenología de la excedencia. Pensamiento y cultura, 13(2), 127-140.

-Xolocotzi, Á. (2005). Fundamento, esencia y Ereignis. En torno a la unidad del camino del pensar de Martin Heidegger. Éndoxa, 20, 733-744.

-Xolocotzi, Á. (2009). 'Quizás el Viejo advierta que le estoy retorciendo el cuello...' Dos décadas de una atormentada relación: Martin Heidegger y Edmund Husserl 1909-1929. Contribuciones desde Coatepec, 15, 11-37.

Sumario: Introducción; 1. El horizonte del planteo sobre excedencia e inapariencia; 2. El abandono del horizonte husserliano; 3. Inapariencia y pensamiento tautológico; 4. Corolarios; Referencias. 